that seeks to improve the reliability of health research reporting.

\author{
Vivian C. McAlister, MD \\ Coeditor, Canadian Fournal of Surgery
}

Competing interests: None declared.

\section{References}

1. Osler W. Men and books. Can Med Assoc 7 1912;2:343-4.

2. Imrie K, Frank JR, Ahmed N, et al. A new era for resident duty hours in surgery calls for greater emphasis on resident wellness. Can 7 Surg 2013;56:295-6.

3. Webber EM, McAlister VC, Gorman L, et al. The past and future of the generalist general surgeon. Can 7 Surg 2014;57:6-7.

4. McAlister V. Consensus ad idem: a protocol for development of consensus statements. Can 7 Surg 2013;56:365.

DOI: $10.1503 /$ cjs.009915

\title{
Élever les normes de production de rapports de recherche chirurgicale pour accroître leur impact
}

es chirurgiens pratiquent patiemment un rituel laborieux et inutile pour obtenir de meilleurs résultats ${ }^{1}$. $\gg$ Cette citation ne se rapportait pas au contrôle de routine de la sécurité chirurgicale, mais plutôt à l'introduction du listérisme par Thomas Roddick à l'Hôpital Général de Montréal. L'observation fut faite par le $\mathrm{D}^{\mathrm{r}}$ William Osler dans un rapport publié dans le fournal de l'Association médicale canadienne $(\mathcal{F} A M C)$, à l'occasion des obsèques, en 1912, de Joseph Lister dans l'abbaye de Westminster. Le $\mathrm{D}^{\mathrm{r}}$ Osler avait conclu en disant : « Pour tout ce qui mérite d'être préservé dans cette vie il y a eu une évolution, mais on n'a pas dévié du grand principe à partir duquel Lister agissait. »

Le $7 A M C$ avait été fondé 1 an plus tôt par la fusion du Montreal Medical fournal et du Maritime Medical News, tous deux établis en 1888. À l'époque, l'industrie de l'édition médicale au Canada était solide et plusieurs revues scientifiques se faisaient concurrence pour un lectorat relativement restreint. Jeune diplômé, le $\mathrm{D}^{\mathrm{r}}$ Osler a publié des articles et des éditoriaux dans des journaux canadiens, auxquels il a continué de contribuer tout au long de sa carrière aux États-Unis et en Angleterre. Son article sur Lister était l'un des 16 articles qu'il publia dans les premières années du $\mathcal{F} A M C$ sous la bannière « Les hommes et les livres ».

Tout comme le New England Fournal of Medicine a été au début le New England Fournal of Medicine and Surgery, les revues canadiennes portaient alors sur les 2 volets de la médecine. Le milieu de l'édition médicale a d'abord été dominé par la chirurgie, jusqu'à ce que les articles médicaux la surpassent au $20^{\mathrm{e}}$ siècle. En 1957, le fournal canadien de chirurgie $(\mathcal{F C C})$ fut fondé pour rétablir l'équilibre. Tout comme le $\mathrm{D}^{\mathrm{r}}$ Osler avait participé à établir les fondations du $7 A M C$, les chefs de file de la chirurgie au Canada publiaient fréquemment des articles dans le $7 C C$. En revanche, on m'a dit récemment que le jeune auteur d'un projet très modeste avait refusé une occasion de publier dans le $\mathcal{F} C$, car à son avis, cela sonnerait le glas de sa recherche. Ce commentaire doit être analysé, et non rejeté.

L'impulsion à l'origine de la création du $\mathcal{F C C}$ était essentiellement protectionniste. Les articles médicaux étaient publiés de préférence dans des revues généralistes parce qu'elles étaient de plus haute qualité. Le $\mathcal{F C C}$ devait être un lieu sûr pour publier des articles en chirurgie, particulièrement du Canada. Pendant un temps, nous avons tenté de rationaliser en disant que la chirurgie nécessite un format de rapport différent de celui de la science médicale. Le facteur d'impact (FI) du $7 C C$ tomba à un nadir de 0,5 ; seulement 1 article publié sur 2 était cité, même 1 seule fois, dans les 2 années suivantes. Pourtant, dès le départ, le $\mathcal{F} C C$ a été produit par des professionnels et la préparation éditoriale était parfaite. Le journal a adopté les améliorations de style à mesure qu'elles ont vu le jour, notamment la normalisation du format pour les références et les unités de mesure. Le journal a aussi adopté des règles au sujet de la qualité d'auteur et participé à des programmes visant à améliorer l'impartialité et à éliminer la fraude. Les articles à faible impact, tels que les rapports de cas ou les essais historiques, ont été refusés. Le nombre de citations générées par les articles du $7 C C$ a quadruplé au cours de la dernière décennie, mais le FI de la revue demeure résolument au second rang parmi les journaux chirurgicaux, à 1,5 . Alors que les journaux chirurgicaux des surspécialités ont tendance à avoir un FI un peu plus élevé que les revues généralistes, le score actuel ne reflète pas la qualité de la chirurgie au Canada.

Au cours des dernières années, le $7 C C$ a fait porter ses efforts sur son impact au Canada plutôt que sur son FI. La collaboration entre les présidents des 
départements de chirurgie, le Collège royal des médecins et chirurgiens du Canada et l'Association médicale canadienne a été ravivée. L'abonnement a été remplacé par un programme pour les établissements universitaires soutenu généreusement par les fondateurs et les sociétés de spécialistes, sous le leadership de l'Association canadienne des chirurgiens généraux. Cette collaboration a permis d'augmenter le tirage et rayonnement : tous les chirurgiens au Canada reçoivent maintenant la revue. Les articles sont annoncés largement sur les médias sociaux et sont disponibles en accès libre immédiat au moyen de liens directs dans les registres scientifiques tels que PubMed et dans les principaux moteurs de recherche, notamment Google. Le $7 C C$ met en évidence les importants développements en chirurgie au Canada, tels que l'impact de la restriction des heures de travail des médecins résidents $^{2}$ ou l'avenir de la chirurgie générale au Canada ${ }^{3}$. Le journal a mis au point un processus d'élaboration et de signalement de consensus ${ }^{4}$.

Pour répondre au défi lancé par notre jeune collègue, le contenu de chaque rapport est le seul facteur qui limite actuellement l'impact des articles publiés dans le
FCC. La prochaine phase de développement consistera donc à élever les normes de production de rapports de recherche chirurgicale. Les rapports auront un impact seulement s'ils sont transparents, précis et convaincants. Le $\mathcal{F C C}$ encouragera la conformité aux directives de présentation de rapports mises de l'avant par le réseau EQUATOR (www.equator-network.org), une initiative internationale qui vise à améliorer la fiabilité des rapports de recherche en santé.

\section{Vivian C. McAlister MD}

Co-rédacteur, Fournal canadien de chirurgie

Intérêts concurrents : Aucuns déclaré

DOI: 10.1503/cjs. 010015

\section{Références}

1. Osler W. Men and books. Can Med Assoc 7 1912;2:343-4.

2. Imrie K, Frank JR, Ahmed N, et al. A new era for resident duty hours in surgery calls for greater emphasis on resident wellness. Can 7 Surg 2013;56:295-6.

3. Webber EM, McAlister VC, Gorman L, et al. The past and future of the generalist general surgeon. Can 7 Surg 2014;57:6-7.

4. McAlister V. Consensus ad idem: a protocol for development of consensus statements. Can 7 Surg 2013;56:365.

\section{Journal canadien \\ Nous croyons au libre accès à la recherche} ${ }^{\text {de }}$ chirurgie

Afin de continuer à assurer le libre accès à tout le contenu du FCS, partout dans le monde, les articles présentés pour publication seront assujettis à compter du $1^{\text {er }}$ janvier 2014 à des frais de soumission de $100 \$$ (dollars canadiens). Les auteurs correspondants affiliés aux commanditaires du $7 C S$ seront exonérés des frais de soumission. Les articles acceptés dans les sections Recherche, Revue et Formation médicale continue sont assujettis à des frais de publication de 700 \$, dans les sections Commentaires et Discussions, à des frais de publication de 500 \$, payables sur acceptation en dollars canadiens.

\section{Les avantages du libre accès}

- Pour les chercheurs et les établissements : visibilité, utilisation et impact accrus de leur travail

- Pour les gouvernements : meilleur rendement de leur investissement dans le financement de la recherche

- Pour la société : des soins aux patients efficaces et efficients, produisant de meilleurs résultats

Les articles du $7 C S$ sont consultables gratuitement sur le site web du Journal (canjsurg.ca) et sur PubMed Central. 